\title{
Detecting Shape Deformation of Soft Objects Using Directional Photoreflectivity Measurement
}

\author{
Yuta Sugiura $^{1 \& 2}$, Gota Kakehi ${ }^{1}$, Anusha Withana ${ }^{1 \& 2}$, Calista Lee $^{1}$, Daisuke Sakamoto $^{2 \& 3}$, \\ Maki Sugimoto $^{1 \& 2}$, Masahiko Inami ${ }^{1 \& 2}$ and Takeo Igarashi ${ }^{2 \& 3}$ \\ ${ }^{1}$ Graduate School of Media Design, \\ Keio University (KMD), \\ 4-1-1 Hiyoshi, Kohoku, \\ Yokohama, 223-8526 Japan \\ ${ }^{2}$ JST, ERATO, IGARASHI \\ ${ }^{3}$ Dept of Computer Science, \\ Design Interface Project, \\ The University of Tokyo, \\ 1-28-1-7F, Koishikawa, Bunkyo, \\ 7-3-1 Hongo, Bunkyo, \\ Tokyo, 112-0002 Japan \\ Tokyo, 113-0033 Japan \\ \{y-sugiura | g.kakehi | anusha | calista | inami\}@kmd.keio.ac.jp, \\ \{sakamoto|sugimoto\}@designinterface.jp, takeo@acm.org
}

\begin{abstract}
We present the FuwaFuwa sensor module, a round, handsize, wireless device for measuring the shape deformations of soft objects such as cushions and plush toys. It can be embedded in typical soft objects in the household without complex installation procedures and without spoiling the softness of the object because it requires no physical connection. Six LEDs in the module emit IR light in six orthogonal directions, and six corresponding photosensors measure the reflected light energy. One can easily convert almost any soft object into a touch-input device that can detect both touch position and surface displacement by embedding multiple FuwaFuwa sensor modules in the object. A variety of example applications illustrate the utility of the FuwaFuwa sensor module. An evaluation of the proposed deformation measurement technique confirms its effectiveness.
\end{abstract}

ACM Classification: H5.m. Information interfaces and presentation (e.g., $\mathrm{HCl}$ ): Miscellaneous.

General terms: Design, Measurement

Keywords: Soft User Interface, Density Measurement, Photoreflectivity, Tangible User Interface, Multiple Sensors

\section{INTRODUCTION}

While traditional input devices such as buttons and handles are made of hard materials, ones made of soft materials are now being introduced for use in interactive systems to provide an emotionally richer user experience $[8,10]$. Soft input devices are especially suitable for children because they are safer than hard ones. However, the sensing techniques used for soft input devices typically require the attachment of electromechanical sensors to soft surfaces to physically measure the deformation. This approach unfortunately spoils the softness of the original material.

In this paper, we present a FuwaFuwa sensor module for detecting shape changes in soft objects. The FuwaFuwa

\footnotetext{
Permission to make digital or hard copies of all or part of this work for personal or classroom use is granted without fee provided that copies are not made or distributed for profit or commercial advantage and that copies bear this notice and the full citation on the first page. To copy otherwise, or republish, to post on servers or to redistribute to lists, requires prior specific permission and/or a fee.

UIST'11, October 16-19, 2011, Santa Barbara, California, USA.

Copyright 2011 ACM 978-1-4503-0716-1/11/10...\$10.00.
}

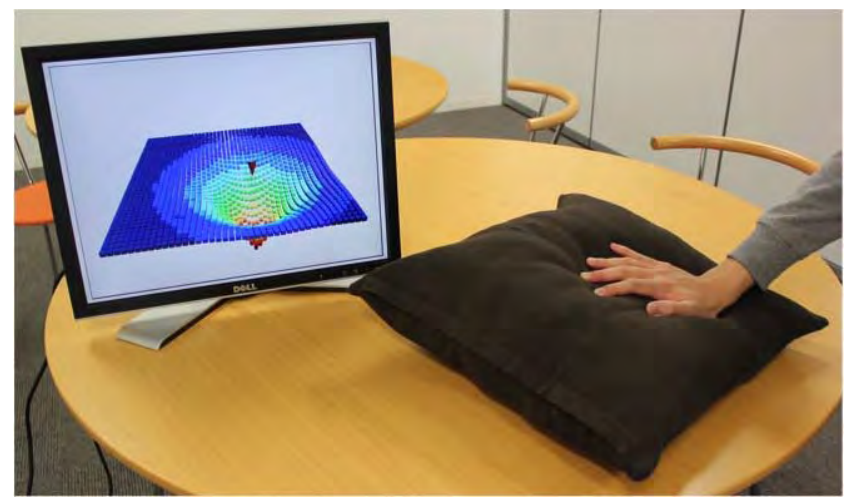

Figure 1: Detecting shape deformation of soft object using directional photoreflectivity measurement

module contains six photoreflectors (each comprising a photosensor and IR light emitter), pointing in six orthogonal directions. The measured photoreflectivities are used to determine the density of the padding material in the object (e.g., cotton and wool).

Our experimental results showed that the photoreflectivity of such materials is a reliable measure for use in determining the density. The user's interactions with a soft object change the shape of the object, resulting in a change in the density of the padding material. The sensors in the FuwaFuwa module detect the degree of this change. As shown in Figure 1, the position of a displacement can be determined by placing several FuwaFuwa modules in the object.

Since it is battery-powered and equipped with wireless communication, the FuwaFuwa sensor module can easily be placed in typical soft objects (e.g. cushions, sofas, pillows, beds) without affecting their softness. This is the main advantage that the FuwaFuwa sensor module has over typical sensors.

The work described in this paper has resulted in four significant contributions to shape deformation detection for soft objects: 
- A method using directional photoreflectivity measurement for detecting pressure changes in soft objects.

- A hand-sized, battery-operated sensor module equipped with wireless communication that can easily be embedded in a soft object without affecting its softness.

- A multi-sensor network system that can detect the barycenter of large soft objects and measure the amount of pressure exerted by the user as input.

- Several example application scenarios for the sensor system.

\section{RELATED WORK}

Many researchers have explored different types of sensing techniques and applications for soft objects as interfaces in today's world.

\section{Plush Toy Interfaces}

The transition from infancy to childhood to adolescence is characterized by certain behaviors, such as remaining attached to a plush toy [26]. Even adults often display an instinctive desire to touch a plush toy. This has led to the recent introduction of interactive plush toys to provide an emotionally richer user experience $[8,10]$. Yonezawa et al. made an interactive plush toy by equipping it with seven types of sensors (bend sensor, microphone, etc.) [28]. They have also created an application to be used with this toy that composes music on the basis of the intensity and frequency of interaction. While their approach preserves the soft properties of the plush toy, installing the sensors is not a simple operation, making its use problematic for typical soft household objects.

\section{Foam Interfaces}

Murakami et al. had developed a device that enabled one to intuitively manipulate 3D CG models [12]. A block of nonconductive urethane foam was cut into a cube, and 90 pieces of conductive urethane foam were attached to it. The resistance of the foam pieces changed in accordance with the deformation of the cube, and the relationship between the resistance and length of the foam pieces was calculated beforehand. In accordance with the lengths recorded for 90 points, the user is able to manipulate the $3 \mathrm{D}$ object by deforming the cube.

Smith et al. developed "Digital Foam" (DF) an input device with which one is able to manipulate a 3D object in a manner similar to the way one manipulates clay [20]. It consists of a number of units created using a combination of conductive cloth and conductive urethane foam. The resistance of each unit changes in accordance with changes in the pressure applied to it. The main difference between DF and FuwaFuwa is that the goal of DF is to create a soft sensor to detect pressure, whereas the FuwaFuwa is a sensor designed to convert existing soft objects into sensing platforms.

\section{Fur-like and Fabric Interfaces}

Various other types of interfaces have been proposed, such as a fur-like interface [2, 4, 13]. Baudisch et al. developed Soap, an input device enclosed in elastic fabric that is based on an optical sensor used for cursor control [1]. The device can be used in various applications, such as for pointing and game control. Although its structure is simple, it cannot measure pressure because the device is made specifically for $\mathrm{x}-\mathrm{y}$ movement.

\section{Elastic Material Interfaces}

Elastic material is a commonly used material in human computer interaction [5, 19, 24, 25]. There have been several attempts at detecting shape deformation using cameras. Sato et al. designed a tabletop system comprising an LCD and an overhead camera for use in detecting the orientation and power of the force [17]. This approach can work well in a limited environment such as a tabletop. However, problems such as camera occlusion occur when it is used in a daily life environment, which is ever-changing.

In the field of robotics, elastic material has been used for developing a robot surface similar to human skin. There have also been several sensors proposed for sensing the touch interaction on the robot's skin $[7,11]$. Kadowaki et al. have developed a method that uses a sensor attached to a soft body to sense and differentiate between interactions (such as push, pinch, and rub) with the object [9]. The soft object used was a piece of urethane foam, with five phototransistors attached at the top and three infrared light-emitting diodes (IR LEDs) attached at the bottom. The electric currents flowing through the phototransistors change in accordance with deformation in the soft body, and these changes are measured and used to determine the amount of deformation. Although this method is sensitive enough to detect very slight changes in deformation, the thickness of the soft body must remain fixed, which makes the method rather inflexible.

\section{Air Pressure Interfaces}

Air itself can create a soft tactile impression. Hiramatsu et al. developed a soft ball-shaped device that reacts to being squeezed, thrown, and shaken [6]. The device has airbagfilled cushions located in a radial fashion at 19 points, and a pneumatic sensor detects the pressure applied. Sponges in the device fill the voids. However, the sensor must be attached to the surface of the device, thus making this approach difficult to apply to many soft objects. Yasumoto et al. proposed a balance-ball-shaped interface as a game controller [27]. Eight pressure sensors attached to the surface of a soft object enable its center of gravity to be measured. As a form of media art, Ueki designed "Tabby," a light that appears to breathe due to the use of an air compressor [22]. A pyroelectric IR sensor inside the lampshade detects the user's touch, but differences in touch pressure are not detected.

\section{Our Interface}

Our FuwaFuwa sensor module has three properties in particular that distinguish it from the sensors described above.

- It is small and wireless, so it can be inserted into simple small objects.

- It can continuously measure pressure. 

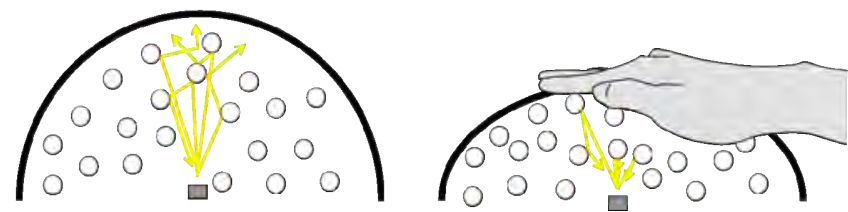

Figure 2: Directionality of reflected light is lower at low density (left) than at high density (right)

- A configuration of such modules can determine the position of touch on a surface.

\section{DIRECTIONAL PHOTOREFLECTIVITY BASED MATERIAL DENSITY MEASUREMENT \\ Principle}

Soft objects such as stuffed toys and pillows usually consist of light materials such as wool, feathers, and cotton as stuffing. The random arrangement and grainy structure of such materials cause incident light to be scattered, resulting in a diffused energy pattern. When these materials are tightly packed (i.e., when the density is increased), they create a considerable amount of directed and specular reflected light. This phenomenon is illustrated in Figure 2.

The IR LED in each photoreflector in the FuwaFuwa sensor module directs a beam towards the stuffing material, and the corresponding photosensor measures the reflected light energy. This measurement indicates the density of the soft material and can be used to detect user interactions with the object.

\section{Relationship between Photoreflectivity and Density}

We conducted an experiment to investigate the relationship between the change in density and the photoreflective properties of soft material. A KODENSHI SG-105 photoreflector (comprising an IR LED and a photosensor) was positioned at the bottom of a clear acrylic box (10.4 x $10.4 \times 14.6 \mathrm{~cm})$ with a cover that could be set at different heights (Figure 3). The box was filled with soft material, the cover height was varied from 12 to $5.2 \mathrm{~cm}$ in intervals of $2 \mathrm{~mm}$, and the light energy reflected on the sensor was measured at each height. The experiment was repeated for three soft materials with different densities: a polyester cotton called "thubuthubushugei-wata” [21], small feathers, and natural cotton (Figure 4).

\section{Experiment Procedure}

1. Place $10.0 \mathrm{~g}$ of soft material in the box.

2. Set the cover to be $12 \mathrm{~cm}$ from the bottom.

3. Record the photosensor output at $1 \mathrm{~Hz}$ for $10 \mathrm{~s}$, collecting a total of ten records.

4. Lower the cover by $2 \mathrm{~mm}$.

5. Repeat steps 3 and 4 until the cover is under the $3.4 \mathrm{~cm}$ from the bottom.

6. Raise the cover by $2 \mathrm{~mm}$.

7. Record the photosensor output at $1 \mathrm{~Hz}$ for $10 \mathrm{~s}$, collecting a total of ten records.

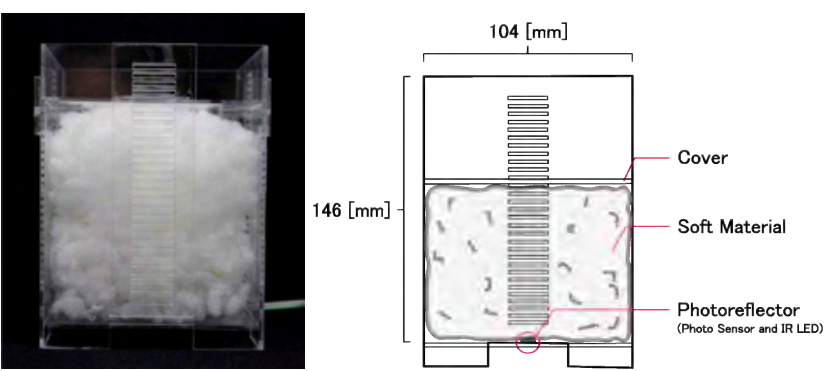

Figure 3: Experimental sensor apparatus

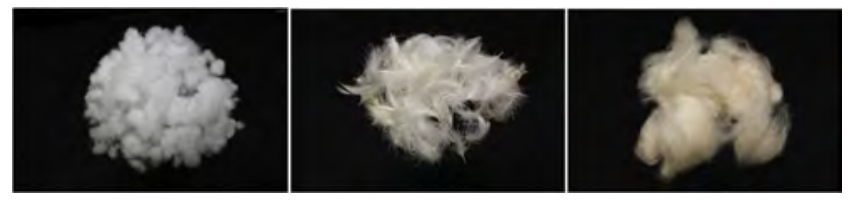

Figure 4: Soft materials: polyester cotton (left), small feathers (center), and natural cotton (right)

8. Repeat steps 6 and 7 until the cover is again at a height of $12 \mathrm{~cm}$ from the bottom.

\section{Experiment Conditions}

Since the density of a cushion is typically about $0.01\left[\mathrm{~g} / \mathrm{cm}^{3}\right]$, we used a density range of 0.0078 to $0.0257\left[\mathrm{~g} / \mathrm{cm}^{3}\right]$ for our experiment. The material was weighed using a Tanita KP103 electronic scale (maximum weight: $120 \mathrm{~g}$, accuracy: 0.1 g), and the photosensor measurements were recorded using an Atmel ATmega328 microcontroller. To check for the effect of hysteresis, we made identical measurements after both lowering and raising the cover. A phototransistor was included in the voltage-divider circuit so that the measured output voltage was inversely proportional to the light reflected on the sensor.

Results

Figure 5 shows a plot of the average measured voltage against the material density for the polyester cotton. The voltage increased with the pressure created by pushing, and it decreased as the pressure was reduced by pulling. But we can observe certain level of hysteresis. Finally, the voltage is saturated at the $0.0257\left[\mathrm{~g} / \mathrm{cm}^{3}\right]$. There was a negligible change in the average measured voltage in the 0.0077 to $0.0092\left[\mathrm{~g} / \mathrm{cm}^{3}\right]$ density range. Therefore, we use polyester cotton density higher than $0.0092\left[\mathrm{~g} / \mathrm{cm}^{3}\right]$.

In the case of the small feathers (Figure 6) and the natural cotton, the voltage is mostly saturated in the measured range, so it is difficult to measure the density of these materials in a cushion using our method. We found that it is possible to measure the density of these materials when the density is low as in feather quilt blanket or down jacket (see Figure 7 and 8). However, we did not use them in our prototype because the weight of the current sensor module is too heavy for such low-density objects. The sensor module might slide according to gravity and cannot stably detect the density.

Other Materials 


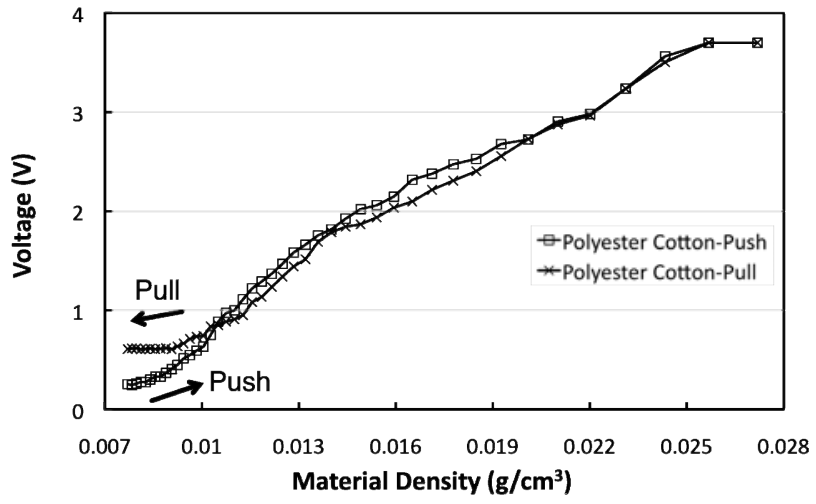

Figure 5: Average measured voltage against material density polyester cotton

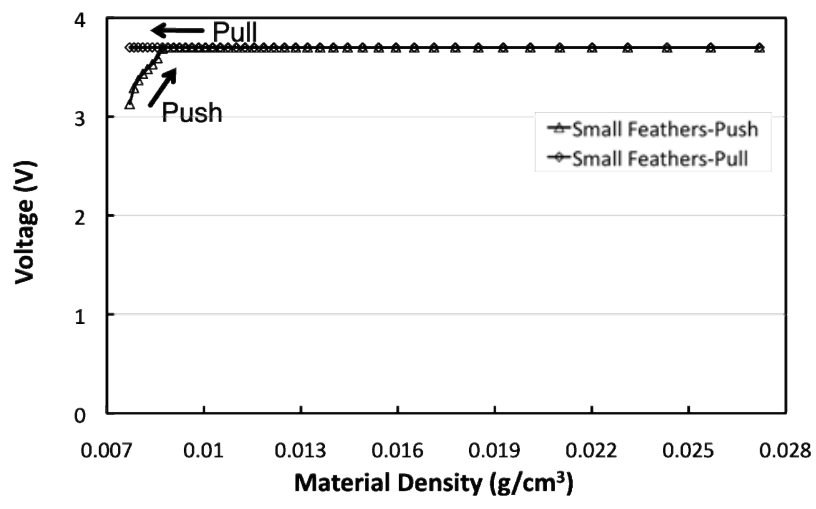

Figure 6: Average measured voltage against material density small feathers

We checked the feasibility of using urethane sponge and polystyrene foam cut at about $\phi 2 \mathrm{~mm}$ as the stuffing material, and found that it was difficult to measure the pressure with such materials because the voltage saturated when they were used.

\section{IMPLEMENTATION}

\section{System Overview}

The hardware configuration used for the prototype implementation is shown in Figure 9. The FuwaFuwa sensor module (or modules) to be installed in various types of soft objects connects wirelessly to a server (MacBook Pro). The server sends data to one or more clients through a TCP/IP network. The clients process the data and display the results.

\section{FuwaFuwa Sensor Module}

Figure 10 shows a photograph of the FuwaFuwa sensor module, and Figure 11 shows the main components. Its six orthogonally arranged photoreflectors enable the energy of the light reflected from each direction to be independently measured. The module requires no physical connection since it is equipped with a wireless communication module (XBee Series1) and a Li-Po battery (3.7 V, $350 \mathrm{~mA}$ ). Its diameter is $64 \mathrm{~mm}$. The cover is made of acrylonitrile butadiene styrene



Figure 7: Average measured voltage against lowlevel density of small feathers

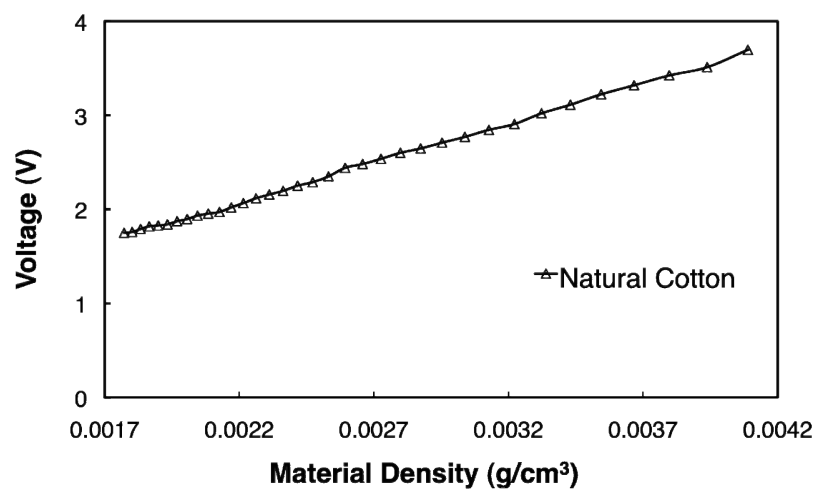

Figure 8: Average measured voltage against lowlevel density of natural cotton

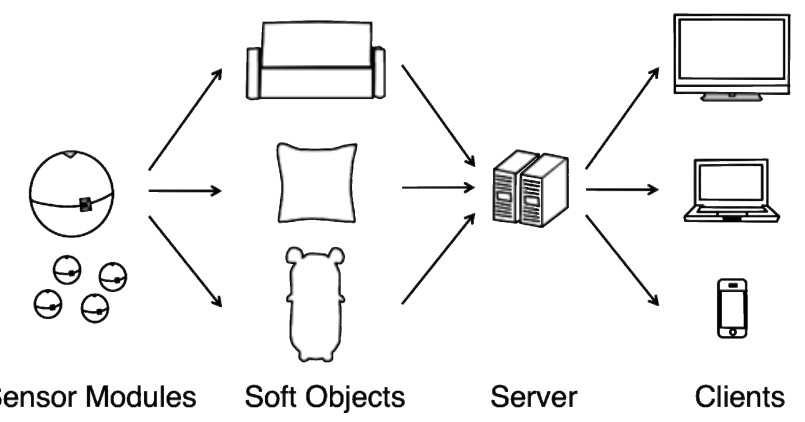

Figure 9: System overview

(ABS) plastic using a 3D printing machine. It can be easily placed in any soft object to create a soft interface.

\section{Data Processing}

An Arduino Pro mini microcontroller (based on ATmega328) is embedded in each FuwaFuwa sensor module for processing the data. It has a 10 -bit A/D converter so that each photoreflector outputs from the value of 0 to 1023, and is connected to the ZigBee module. Since an ID number can be assigned to each ZigBee module, multiple sensor modules can be used at the same time. 


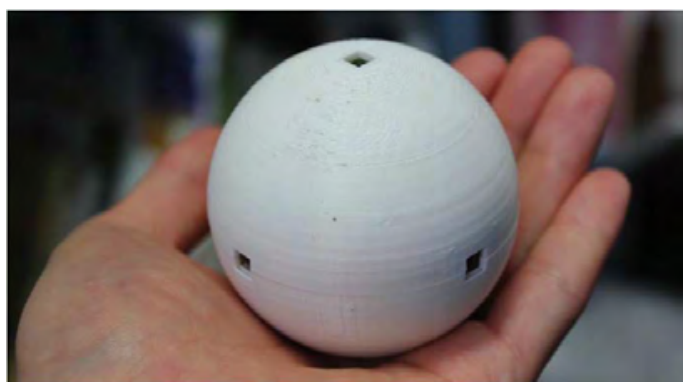

Figure 10: Photograph of FuwaFuwa sensor module ( 3 of the 6 photoreflectors are visible)

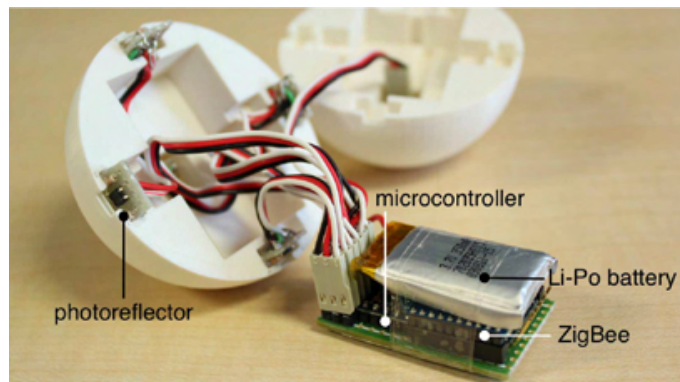

Figure 11: Main components of FuwaFuwa sensor module

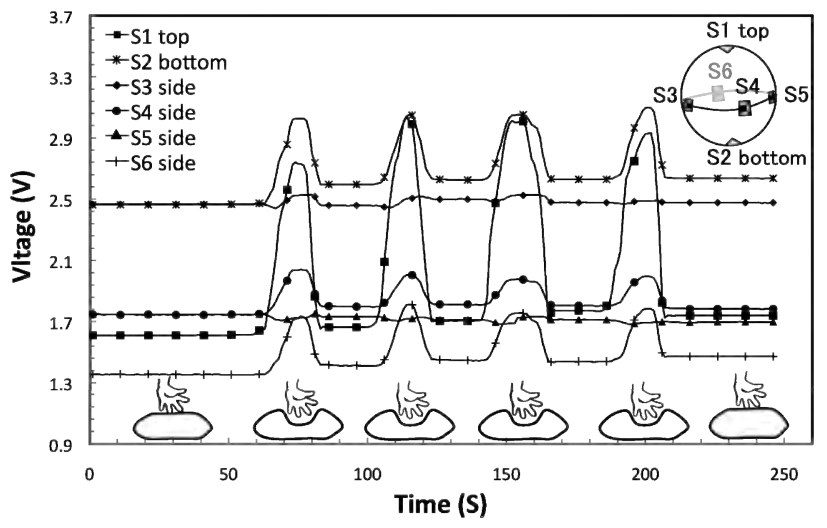

Figure 12: Each sensor values in the module

\begin{tabular}{c|c|c|c} 
2 Bytes & 1 Byte & 1 Byte & $2 \times$ N Bytes \\
\hline START & Module ID & $\begin{array}{c}\text { No. of Photore- } \\
\text { flector in the } \\
\text { module }\end{array}$ & $\begin{array}{c}\text { Photoreflector } \\
\text { Value }\end{array}$ \\
&
\end{tabular}

Table 1: User datagram protocol for server-client connection

Figure 12 shows the individual sensor readings when FuwaFuwa is embedded in a spherical soft object and the object is pushed along the axis of sensor 1 and 2 (S1 and S2) towards $\mathrm{S} 1$. According to the graph, S1 has the highest voltage variations and S2 has the second highest variations due to the directionality of the density variation inside the object.

\section{Proxy Server}

A proxy server connects the FuwaFuwa sensor module with the client software using serial communication at 57,600 bps.

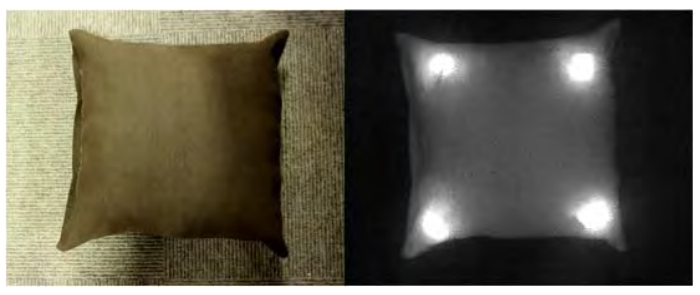

Figure 13: Detecting locations of FuwaFuwa sensor modules: image with normal camera (left) and IR camera (right)

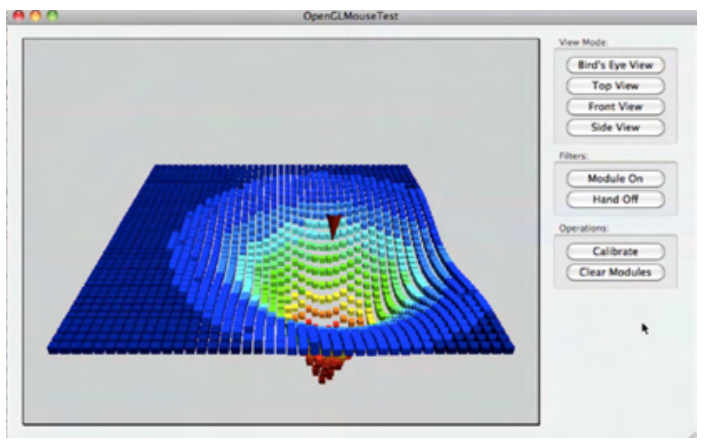

Figure 14: Visualized sensor data

The server processes the received data and sends it to one or more clients using the user datagram protocol (Table 1).

\section{Multiple FuwaFuwa Sensor Modules}

By installing multiple FuwaFuwa sensor modules in a larger soft object, collected sensor measurements can be used to indentify the point of touch on the surface. This means that any soft object can be converted used as a touch-sensitive surface. Moreover, it enables the strength of the touch to be measured, which is difficult with a hard touch surface. Next we explain the method for calibrating the module locations, the method for calculating the touch point, and the software used for visualizing the sensor data.

\section{Module Location Calibration}

When multiple sensor modules are placed in an object, their locations must be calibrated before they can be used. The LED in each module emits IR light in a particular direction. An IR camera can detect these high-intensity light beams even through the object's stuffing and covering, as shown in Figure 13. When a sensor-embedded object is placed within the camera's field of view, the user can specify the location of the embedded sensors by clicking on them in the software.

\section{Barycenter Calculation}

The touch point is detected by calculating the barycenter of the sensor modules using the calibrated sensor locations and the photorefractive values. The coordinates on the soft object are the same as the coordinates that are calibrated using the IR camera. This means that the user's input will become the barycenter of the calibrated coordinate by the module location calibration process. The system detects 1) two- 
dimensional point and 2) pressure level of user's pressing gesture. The formula is:

$$
\begin{aligned}
& M=\sum_{i=1}^{n} p_{i} \\
& x=\left(\sum_{i=1}^{n} p_{i} x_{i}\right) / M \\
& y=\left(\sum_{i=1}^{n} p_{i} y_{i}\right) / M
\end{aligned}
$$

where $n$ is the number of sensor modules. $p_{i}$ is the pressure that is the total value of photoreflector values at sensor module $i$. $x_{i}$ and $y_{i}$ represent the $\mathrm{x}-\mathrm{y}$ coordinates of sensor $i . M$ is total value of pressure, and will be used as the pressure level.

\section{Data Visualization}

We designed software for visualizing the sensor data based on the pressure widgets developed by Ramos et al. [15]. The color and shape of the displayed image change with changes in pressure (Figure 14). The image can be viewed from various viewpoints such as the top, side and front.

\section{EXAMPLE APPLICATIONS}

To help illustrate the utility of the FuwaFuwa sensor module, we present several example domains where the technology could be deployed.

\section{Music Instrument}

We converted a cushion into a music composer using FuwaFuwa (Figure 15). Two sensors were embedded in the either side of the cushion and it can detect the touch position and applied pressure. Keys are shown in different colors (projected) and the pitch of each key can be changed using the amount of pressure applied. Multiple cushions can be combined to make a multi-user music composer application.

\section{Soft Interactive Robot}

Many researchers have been exploring the usage of soft robots because softness would help to reduce the psychological distance between robots and humans $[16,23]$. With this in mind, we developed a soft pet robot named "sofmo" using one FuwaFuwa sensor module. The robot detects user actions and reacts accordingly. The robot changes its voice in accordance with changes in touch pressure (Figure 16) and moves its eyes (actuated by servo motors) towards the touch position (Figure 17). It can thus support physical interactions such as hitting, throwing, and hugging, and can be used as a safe robotic toy for children or to provide therapy for elderly people. Additionally, robots located remotely can be synchronized to provide a new form of communication media [18].

\section{Gaming Peripherals}

We installed a FuwaFuwa sensor module in a small spherical cushion to create a game controller for a simple game (Figure 18). The user controls the game character by manipulating

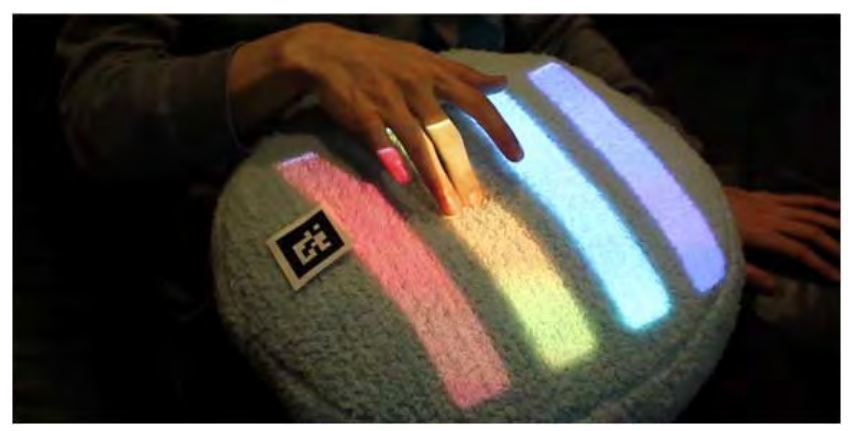

Figure 15: Music instrument
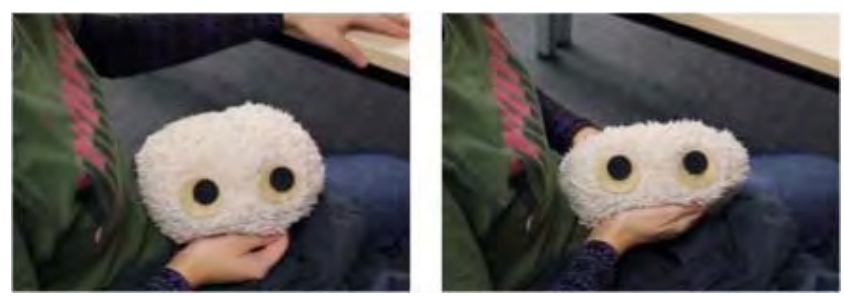

Figure 16: Voice changes with change in touch pressure
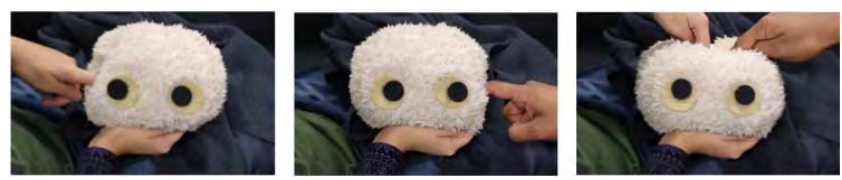

Figure 17: Eyes move toward touch position

the surface of the cushion. Different actions such as squeeze, hit, push, and rub are used to trigger different actions.

We also developed an entertainment application-a balancing game. An ice floe carrying some penguins tips when the user shifts his/her center of gravity on the beanbag chair, which used as the game controller (Figure 19). The size of the beanbag chair is $1 \mathrm{~m}$ in diameter, and four sensor modules in the chair are used to measure the axis of the center of gravity of the user. The FuwaFuwa sensor module can also be applied to health-related games incorporating body movements [14, 27].

\section{Domestic Media Controller}

Soft objects are commonly found in home environments. We created a media controller application for a living room by converting a cushion into a remote controller for a digital home theatre system. Three FuwaFuwa sensor modules are installed in the cushion, and a media control menu is projected onto the cushion (Figure 20). The user pushes the cushion in the appropriate places to select the desired functions. 


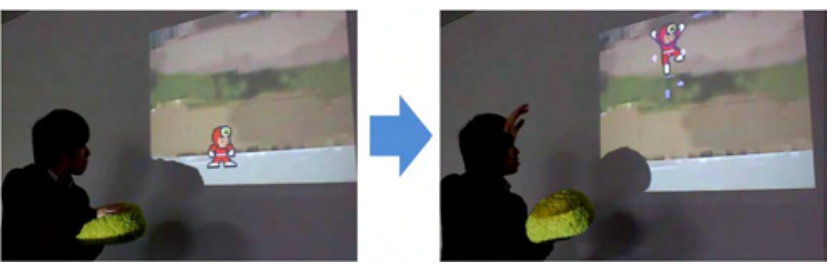

Figure 18: Game controller

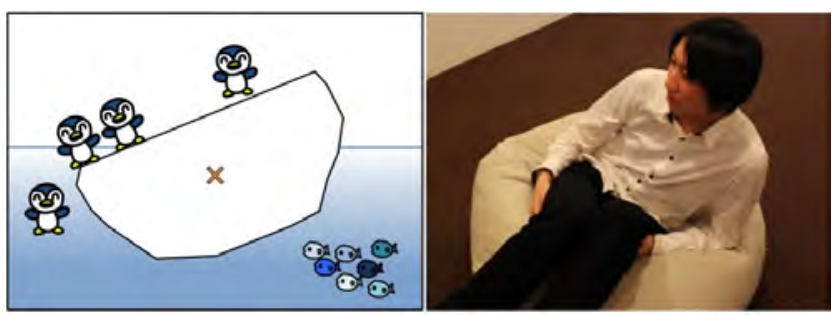

Figure 19: Balancing game display (left); beanbag chair as game controller (right)

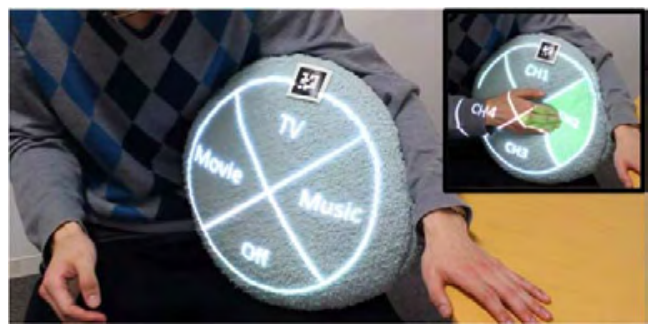

Figure 20: Media controller

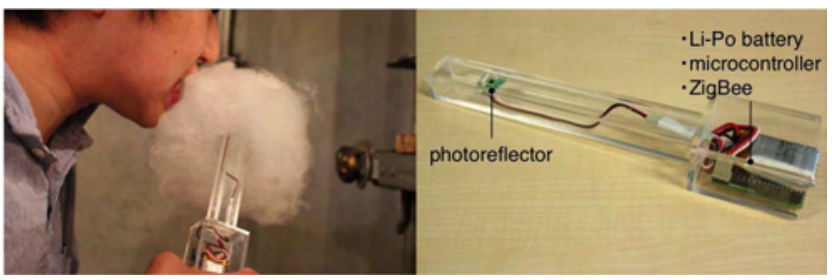

Figure 21: Stick for holding candy floss

\section{Entertainment Candy Floss}

Candy floss (or cotton candy) has properties very similar to those of cotton. We attached a photoreflector to a stick holding candy floss (Figure 21). The stick (made from acrylic) was embedded with a microcontroller, wireless module, and a small battery. The stick detects when the user eats the floss and responds by playing a sound.

These are but a few examples of possible usages, showing how the FuwaFuwa sensor module can be used to enhance our daily life in a pleasurable manner.

\section{Human Activity Sensors}

Because the FuwaFuwa sensor module is small, it can be installed in such household objects as sofas, pillows, and beds. Moreover, it can detect a person's behavior and condition [3]. For example, a module installed in a pillow was used to detect a user's behavior. When the user falls asleep, the surrounding lights are automatically dimmed.

\section{DISCUSSION}

Advantages

The FuwaFuwa sensor module has three important advantages over existing technologies for soft interfaces. Firstly, it does not spoil the softness of the object into which it is embedded, mainly because it requires no physical connection and is relatively small. Secondly, it can detect a wide range of variations in deformation. As shown in the experimental results, it can detect gradual changes in polyester cotton density and thus different deformation levels. Finally, it can be easily installed in almost any soft object because no physical connections are required. We believe that these features of the FuwaFuwa sensor module enable it to be used to create powerful and economical ambient sensor environments for household applications.

\section{Accuracy}

Overall, the system recognized the user input (points and pressure levels) on the soft object's surface accurately. In the media controller application, which provides four selections on the object's surface, the system recognized every user selection with four sensor modules. In the game controller application, the user could select various types of shotguns by adjusting the pushing time. However, there were cases of incorrect operations. In one case, for example, lengthy use shifted the orientation of the modules in the object, which distorted their calibration. In another case, the cotton stuffing clogged up a photoreactor opening.

\section{Limitation and Future Work}

Our FuwaFuwa sensor module is a "proof-of-concept" implementation, so that it has some hardware limitations. The Li-Po battery can supply power for only up to 3 hours, after which the user has to remove and charge the battery. Because the sensor module is installed within a soft object, removing it to charge the battery is inconvenient, especially when it is located deep in the object such as a beanbag chair. Also, its diameter is $64 \mathrm{~mm}$, meaning that it cannot be installed it in very small and/or thin objects such as small plush toys, comforters, rugs, and clothing. There is thus a trade-off between available usage time and module size (current battery, $350 \mathrm{mAh}$ Li-Po battery 36mm vs. smaller 110mAh 28mm). The current implementation of FuwaFuwa uses a generalpurpose microcontroller (arduino) and a commercial ZigBee module. In a more customized implementation however, we can select components with smaller footprint and integrate them into a more power-efficient system with smaller form factor. For larger objects such as beanbag chairs that are not normally moved, an external power supply might be more appropriate. Furthermore, we plan to use power-saving mechanisms such as activity detection using an accelerometer or a single IR sensor, power hopping, and so on. Currently, the sensor's cover is made of hard plastic. In the future, we plan to use soft materials such as polyurethane rubber.

An IR camera is used to calibrate the module locations. However, fabrics that are tightly woven or are heavy (such as velour and leather) block IR light. Moreover, if the modules 
are installed deep in the soft material, it is hard for the IR camera to recognize the IR light. Additionally, having to hold the camera over the object to capture the image is inconvenient, intrusive, and troublesome for the user. A better calibration method would be to have the modules detect their location automatically simply by having the user touch the object a few times.

\section{CONCLUSION}

We have developed a sensor module that enables a user to interact directly with soft objects. Our FuwaFuwa sensor module can be embedded in typical soft objects in the household without complex installation procedures and without spoiling the softness of the object because it requires no physical connection. Using a directional, photoreflectivitybased shape deformation measurement technique, it can detect a wide range of deformations. A variety of example applications illustrate the utility of the FuwaFuwa sensor module. An evaluation of the proposed deformation measurement technique confirmed its effectiveness.

\section{REFERENCES}

1. Baudisch, P., Sinclair, M. and Wilson, A. Soap: a pointing device that works in mid-air. In Proc. UIST '06, ACM (2006), pp. 43-46.

2. Chang, J., MacLean, K. and Yohanan, S. Gesture Recognition in the Haptic Creature. In Proc. EuroHaptics '10, pp. 385-391.

3. Choe, E. K., Kientz, J. A., Halko, S., Fonville, A., Sakaguchi, D. and Watson, N. F. Opportunities for computing to support healthy sleep behavior. In Ext. Abst. CHI '10, ACM (2010), pp. 3661-3666.

4. Furukawa, M., Uema, Y., Sugimoto, M. and Inami, M. Fur interface with bristling effect induced by vibration. In Proc. AH '10, ACM (2010), Article 17, 6 pages.

5. Harrison, C. and Hudson, S. E. Providing Dynamically Changeable Physical Buttons on a Visual Display. In Proc. CHI '09, ACM (2009), pp. 299-308.

6. Hiramatsu, R. Puyo-con. In ACM SIGGRAPH ASIA 2009 Art Gallery \& Emerging Technologies, ACM (2009), pp. 81-81.

7. Inaba, M., Hoshino, Y., Nagasaka, K., Kagami, S. and Inoue, $\mathrm{H}$. A full-body tactile sensor suit using electrically conductive fabric and strings. In Proc. IROS '96, IEEE (1996), pp. 450-457.

8. Johnson, M. P., Wilson, A., Blumberg, B., Kline, C. and Bobick, A. Sympathetic interfaces: using a plush toy to direct synthetic characters. In Proc. CHI '99, ACM (1999), pp. 152-158.

9. Kadowaki, A., Yoshikai, T., Hayashi, M., Inaba, M. Development of Soft Sensor Exterior Embedded with Multiaxis Deformable Tactile Sensor System. In Proc. Ro-Man '09, IEEE (2009), pp.1093-1098.

10. Marti, S. and Schmandt, C. Physical embodiments for mobile communication agents. In Proc. UIST '05, ACM (2005), pp. 231-240.
11. Miyashita, T., Tajika, T., Ishiguro, H., Kogure, K. and Hagita, N. Haptic communication between humans and robots. In Proc. ISRR '05, 2005.

12. Murakami, T. and Nakajima, N. Direct and intuitive input device for 3-D shape deformation. In Proc. CHI '94, ACM (1994), pp. 233-236.

13. Nakajima, K., Itoh, Y., Yoshida, A., Takashima, K., Kitamura, Y. and Kishino, F. FuSA2 touch display. In ACM SIGGRAPH 2010 Emerging Technologies, ACM (2010), Article 11, 1 pages.

14. Nintendo, Wii Fit, http://www.nintendo.co.jp/wii/rfnj/

15. Ramos, G., Boulos, M. and Balakrishnan, R. Pressure widgets. In Proc. CHI '04, ACM (2004), pp. 487-494.

16. Samani, H. A., et al. A Design Process for Lovotics. Human - Robot Personal Relationships. In Proc. HRPR '10, Springer LNICST series, Volume 59, pp. 118-125.

17. Sato, T., Mamiya, H., Koike, H. and Fukuchi, K. Photoelastictouch: transparent rubbery tangible interface using an lcd and photoelasticity. In Proc. UIST '09, ACM (2009), pp. 43-50.

18. Sekiguchi, D., Inami, M. and Tachi, S. RobotPHONE: RUI for Interpersonal Communication. In Ext. Abst. CHI '01, ACM (2001), pp. 277-278.

19. Slyper, R., Poupyrev, I. and Hodgins, J. Sensing through structure: designing soft silicone sensors. In Proc. TEI '11, ACM (2011), pp. 213-220.

20. Smith, R. T., Thomas, B. H. and Piekarski, W. Digital foam interaction techniques for 3D modeling. In Proc. VRST '08, ACM (2008), pp. 61-68.

21. Thubuthubu-shugei-wata, Arai Corporation, http://www.sofiapack.com/hobby.html

22. Ueki, A., Kamata, M. and Inakage, M. Tabby: designing of coexisting entertainment content in everyday life by expanding the design of furniture. In Proc. ACE '07, ACM (2007), pp. 72-78.

23. Wada, K. and Shibata, T. Social effects of robot therapy in a care house - change of social network of the residents for two months. In Proc. ICRA '07, IEEE (2007), pp. 1250-1255.

24. Weinberg, G. Orth, M. and Russo, P. The embroidered musical ball: a squeezable instrument for expressive performance. In Ext. Abst. CHI '00, ACM (2000), pp. 283284.

25. Weiss, M., Wagner, J., Jansen, Y., Jennings, R., Khoshabeh, R., Hollan, J. D. and Borchers, J. 2009. SLAP widgets: bridging the gap between virtual and physical controls on tabletops. In Proc. CHI '09, ACM (2009), pp. 481-490.

26. Winnicott, D. (1953). Transitional objects and transitional phenomena, Int. J. Psychoanal, 34, pp. 89-97.

27. Yasumoto, M. Balance ball interface. In $A C M$ SIGGRAPH ASIA 2008 Art Gallery \& Emerging Technologies, ACM (2008), pp. 41-41.

28. Yonezawa, T., Clarkson, B., Yasumura, M. and Mase, K. Context-aware sensor-doll as a music expression device. In Ext. Abst. CHI ’01, ACM (2001), pp. 307-308. 